\title{
Acessibilidade como um Valor no Projeto de Tecnologias Educacionais: Uma Avaliação do Sistema de Apoio à Realização do ENEM para Surdos
}

\author{
Maíra Codo Canal $^{1}$, Deógenes Junior ${ }^{1}$, Laura Sánchez García ${ }^{1}$, Roberto Pereira ${ }^{1}$ \\ ${ }^{1}$ Departamento de Informática - Universidade Federal do Paraná (UFPR) \\ Caixa Postal 19.081 - 81.531-980 - Curitiba - PR - Brasil \\ \{mairapdn, deogenesj\}@gmail.com, \{laura, rpereira\}@inf.ufpr.br
}

\begin{abstract}
Although research on accessibility in computing systems is a topic of traditional interest in Human-Computer Interaction and Informatics in Education, the dissemination of computing technology has required considering accessibility in an increasingly comprehensive and systemic way. This paper proposes that accessibility in computing systems must be understood as a human value, and traditional methods of interface design and evaluation must be revisited to cope with such understanding throughout the entire design process. This paper presents a practical study of accessibility evaluation for the deaf in the environment to support High School Exam for Brazilian students provided by the National Institute for Educational Studies and Research on Youtube. Results point out to accessibility problems in videos, texts, images and personalization by users, which, from the perspective of values, can trigger negative individual and social consequences in aspects of autonomy, economics, identity and equality that can not be reversed or corrected only by technical updates.
\end{abstract}

Resumo. Embora pesquisas sobre acessibilidade em sistemas computacionais sejam um tema de interesse em Interação Humano-Computador (IHC) e Informática na Educação, a disseminação de tecnologias computacionais para muitos aspectos possiveis da vida individual e em sociedade tem exigido considerar a acessibilidade de uma forma cada vez mais abrangente e sistêmica. Este artigo propõe que a acessibilidade em sistemas computacionais seja entendida como um valor humano, e que métodos tradicionais de design e avaliação de interfaces sejam revisitados para apoiar esse entendimento de modo transversal a todo o processo de design, da concepção ao uso e descarte da solução projetada. Este artigo apresenta um estudo prático de avaliação de acessibilidade para surdos no ambiente de apoio à realização do Exame Nacional do Ensino Médio (ENEM) disponibilizado pelo Instituto Nacional de Estudos e Pesquisas Educacionais (INEP) no Youtube. Resultados indicam problemas de acessibilidade em vídeos, com textos, imagens, e personalização pelos usuários que, sob a perspectiva de valores, podem desencadear consequências individuais e sociais negativas em aspectos de autonomia, econômicos, identidade e igualdade que não podem ser revertidas ou corrigidas somente com a atualização técnica do sistema. 
VII Congresso Brasileiro de Informática na Educação (CBIE 2018)

Anais do XXIX Simpósio Brasileiro de Informática na Educação (SBIE 2018)

\section{Introdução}

Sistemas computacionais influenciam as relações humanas e mediam a comunicação, permeando a vida individual e coletiva, sendo usados em variados ambientes, como os de trabalho, saúde, educação, etc. À medida que a tecnologia vem estando mais presente na vida das pessoas, as oportunidades e os desafios apresentados pelo seu uso exigem que métodos tradicionais IHC sejam repensados e que novos conceitos como valores humanos sejam considerados. Apesar de serem considerados importantes, valores ainda são deixados às margens no projeto de sistemas computacionais [Leitão et al. 2017].

Pereira et al. (2018) se fundamentam na Semiótica [Peirce 1955] e na Teoria de Affordances [Gibson 1979] para considerar que valor é alguma coisa que denota importância para alguém em algum respeito ou capacidade. Nessa direção, entender uma perspectiva orientada a valores no projeto de tecnologias é levar em consideração não somente os aspectos da interação dos usuários com a tecnologia, mas, também, as demais relações existentes entre o indivíduo e o mundo em que vive. Dessa maneira, valores transcendem aspectos de acessibilidade, privacidade, autonomia, exclusão, etc.

Entender acessibilidade como um valor humano carrega necessariamente o entendimento de acessibilidade como um direito da pessoa e implica reconhecer que toda pessoa pode ser ou estar deficiente de acordo com as características do contexto, devendo ser beneficiada por projetos, produtos e práticas que primem pela acessibilidade para todos. Colocar esse entendimento em prática no design de sistemas interativos requer a consideração de questões informais, formais e técnicas de modo articulado e sistêmico, demandando por recursos que apoiem os profissionais envolvidos nessa tarefa [Pereira et al. 2013].

Em 2017, o Ministério da Educação (MEC) implantou o uso de sistemas computacionais para apoio aos surdos na realização da prova do ENEM $^{1}$, exame cujo resultado é considerado para o ingresso às universidades públicas no Brasil. Portanto, é compulsório que estes ambientes sejam adequados ao uso pelos surdos, de maneira a não existir barreiras para o acesso desse cidadão ao conhecimento disponibilizado. Investigar a acessibilidade de tais recursos é essencial para se garantir o acesso universal do cidadão ao conhecimento, citado como o Grande Desafio 4 da Sociedade Brasileira de Computação [Baranauskas e Souza 2006].

Entender acessibilidade como um valor é especialmente crítico em contextos nos quais as barreiras introduzidas pela tecnologia (ou geradas pela falta de acesso a elas) aprofundam a desigualdade de oportunidades e catalisam desigualdades socioeconômicas. Embora a negligência com acessibilidade seja preocupante em qualquer contexto, ela é particularmente prejudicial no projeto de tecnologias educacionais, tanto por impactarem diretamente na vida social dos estudantes, quanto por influenciarem as práticas de ensino e aprendizagem dos envolvidos.

O entendimento e prática em uma perspectiva orientada a valores, porém, implica em ajustes conceituais e práticos que são especialmente desafiadores para profissionais com formação em Computação, que comumente não possuem formação em questões básicas de qualidade em IHC. Para discutir essa perspectiva em um

\footnotetext{
${ }^{1}$ Portal MEC. http://portal.mec.gov.br/ultimas-noticias/418-enem-946573306/52141-candidatosdeficientes-auditivos-farao-videoprovas-com-libras
} 
VII Congresso Brasileiro de Informática na Educação (CBIE 2018)

Anais do XXIX Simpósio Brasileiro de Informática na Educação (SBIE 2018)

contexto prático e socialmente relevante, este artigo apresenta resultados da avaliação de acessibilidade para surdos no ambiente de apoio a realização do ENEM por meio de vídeos disponibilizados pelo INEP no Youtube. A avaliação foi realizada por especialistas em IHC com base em um conjunto de recomendações para surdos em tecnologias educacionais [Canal e García 2015]. Os resultados apontaram problemas relacionados ao uso de vídeos, imagens, textos e personalização pelo usuário. $\mathrm{O}$ artigo discute os resultados dessa avaliação adotando uma perspectiva orientada a valores que mostra aspectos que seriam facilmente ignorados caso uma perspectiva centrada em aspectos técnicos fosse adotada.

\section{Revisão bibliográfica}

Acessibilidade pode ser definida como a capacidade de atender, de maneira satisfatória, à diversidade de usuários, com habilidades, preferências, necessidades e diferentes limitações cognitivas e motoras [Santana et al. 2009]. Apesar de esse conceito ter sido adotado em pesquisas sobre acessibilidade em sistemas computacionais, Pereira et al. (2013) apontam para a necessidade de se considerar a acessibilidade além de um aspecto referente à interação do usuário com a tecnologia, como um requisito de qualidade de software e, assim, considerando também o contexto social. Nessa direção, Pereira e Baranauskas (2016) sugerem que problemas de interação do usuário em sistemas interativos não possam ser discutidos e solucionados por meio de uma visão centrada em questões técnicas, ignorando o mundo social no qual os sistemas são usados e os indivíduos vivem. Para os autores, os impactos que os sistemas de informação têm desencadeado em aspectos econômicos, políticos, éticos e na vida em sociedade estão cada vez mais evidentes. Contudo, a adoção de uma visão centrada em questões técnicas como prática padrão é causada parcialmente pela falta de conhecimento e ferramental de apoio a uma visão sistêmica e socialmente consciente.

$\mathrm{Na}$ educação, tecnologias têm sido aplicadas para diferentes finalidades. A informatização do processo de avaliação, por exemplo, tem o potencial de facilitar procedimentos lentos e tediosos envolvidos no projeto, na entrega e na análise da avaliação [Beech 2011]. A avaliação baseada em computador (CBA, do inglês Computer-Based Assessment) é uma iniciativa nesse contexto e pode ser composta de diferentes tipos de questões, como verdadeiro/falso, múltipla escolha, dissertação, etc.

Estudos da literatura, como os de Luephattanasuk et al. (2011) e Beech (2011), sugerem que o uso da CBA pode trazer benefícios aos professores e estudantes, mas que para isso é necessário levar em consideração a acessibilidade de tais recursos de maneira que todos possam se beneficiar desse tipo de avaliação, incluindo aqueles com deficiências. Luephattanasuk et al. (2011) afirmam que recomendações mais específicas são necessárias para identificar problemas de acessibilidade para que pessoas com deficiência possam interagir sem barreiras com os tipos de questões da CBA. Nessa direção, é necessário assegurar que a CBA possa ser utilizada por todos, sem exclusão, e para isso são necessários métodos que apoiem a avaliação e correção dos problemas.

No contexto dos surdos, que têm como língua preferencial a língua de sinais, Bueno et al. (2007) afirmam que o uso de Ambientes Virtuais de Aprendizagem (AVAs) pode ser apropriado como apoio aos processos de ensino-aprendizagem, sendo a avaliação parte constituinte desses ambientes. No entanto, esse benefício só pode ser explorado se os recursos computacionais estiverem devidamente adaptados. Canal e García (2014) investigaram a acessibilidade dos tipos de questões oferecidas pelo 
VII Congresso Brasileiro de Informática na Educação (CBIE 2018)

Anais do XXIX Simpósio Brasileiro de Informática na Educação (SBIE 2018)

Moodle no contexto dos surdos, e identificaram problemas no uso de vídeos, imagens, textos e personalização pelo usuário que não seriam cobertos por diretrizes genéricas de acessibilidade na Web

No Brasil, o MEC tem incentivado o uso de ambientes computacionais para apoio à preparação do ENEM $^{2}$ Entretanto, trabalhos como os de Canal et al. (2017), mostraram que esses ambientes possuem problemas de acessibilidade no contexto dos surdos que causam barreiras e até mesmo o impedimento do acesso para esse público. Entendendo acessibilidade como um valor, questões que vão além de aspectos técnicos, como os atributos de qualidade do software, da relação dos estudantes com a tecnologia, à aspectos da vida individual e social devem ser levadas em consideração no projeto e avaliação desses ambientes antes que eles sejam disponibilizados para uso.

De acordo com Pereira et al. (2018), a tecnologia permeia aspectos da vida individual e social das pessoas não somente na automatização das atividades e na resolução de problemas, mas também na conexão, na mediação de interações sociais diversas e complexas, no entretenimento, criatividade, aprendizado, entre outros. Nesse sentido, a investigação sobre o uso da tecnologia não pode mais ser focada somente nas questões técnicas como normalmente ocorre nos métodos e técnicas de avaliação em IHC, mas deve, também, levar em consideração os ambientes em que os indivíduos vivem e as diversas possibilidades de uso e efeitos que as tecnologias podem desencadear na vida das pessoas.

\section{Método}

O método utilizado neste trabalho se baseia em um conjunto de recomendações de acessibilidade para surdos proposto por Canal e García (2015), para apoiar a avaliação dos tipos de questões usadas na CBA em AVAs. A partir dessas recomendações, dois especialistas em IHC realizaram uma avaliação de acessibilidade para surdos nos tipos de questões usadas nos vídeos disponibilizados pelo INEP para apoio à realização do ENEM (e.g., dissertação e múltipla escolha). Um dos especialistas é pesquisador de acessibilidade para surdos e Informática na Educação, e já estava familiarizado com a aplicação das recomendações. O outro tinha conhecimentos sobre avaliação de usabilidade. A avaliação dos tipos de questões foi realizada considerando a tarefa da elaboração das respostas pelos estudantes.

Videoprova do ENEM. O sistema de apoio à realização da prova do ENEM, denominado "videoprova", oferece uma série de vídeos gravados com a interpretação em Língua brasileira de sinais (Libras) das orientações, dos enunciados das questões e das alternativas de resposta. A videoprova compreende também um sistema de navegação interativa dos vídeos, bem como funcionalidades de controle do vídeo baseadas em ícones. Cada estudante surdo, que fez a solicitação do uso da tecnologia no momento da inscrição para o exame, recebeu um notebook com acesso à videoprova. Os estudantes deveriam marcar as respostas das questões no cartão-resposta e na folha de redação em papel $^{3}$. Devido a esse fato, para a avaliação, a videoprova foi analisada pelos

\footnotetext{
${ }^{2}$ Brasil Gov. http://www.brasil.gov.br/editoria/educacao-e-ciencia/2014/08/saiba-como-estudar-para-oenem-pela-internet

${ }^{3}$ Portal do MEC. http://portal.mec.gov.br/component/tags/tag/44091
} 
VII Congresso Brasileiro de Informática na Educação (CBIE 2018)

Anais do XXIX Simpósio Brasileiro de Informática na Educação (SBIE 2018)

especialistas apenas por meio de vídeos que foram disponibilizados pelo (INEP) no Youtube, e que continham todas as questões do $\mathrm{ENEM}^{4}$.

Recomendações. O conjunto de recomendações proposto por Canal e García (2015) é formado por 34 recomendações organizadas em nove categorias (i.e., "Vídeo em Língua de Sinais", "Apresentação Alternativa para Áudio", "Apresentação Alternativa para Texto Escrito", "Apresentação Alternativa para Conteúdo Visual", "Conteúdo", "Navegação", "Dicionários e Glossários de Termos", "Colaboração entre os Estudantes" e "Feedback"), no qual as recomendações são aplicadas de acordo com 6 tipos de questões (i.e., "múltipla escolha", "verdadeiro/falso", "associação", "lacuna", "resposta curta" e "dissertação") e sua aplicação em perguntas e respostas. Para cada recomendação há três perfis (professor, estudante e desenvolvedor) com sugestões para auxiliar na sua aplicação. $O$ objetivo dessas recomendações é prover suportes computacionais acessíveis dos tipos de questões usadas na CBA em AVAs para surdos. No ENEM, devido ao fato de o estudante responder as questões no papel, algumas recomendações de certas categorias não são passíveis de aplicação, como as das categorias: "Dicionários e Glossários de Termos", "Apresentação alternativa para áudio", "Colaboração entre os Estudantes" e "Feedback".

Procedimentos da avaliação. Para realizar a avaliação, os dois especialistas separadamente acessaram a videoprova do ENEM disponibilizada pelo INEP por meio de vídeos no Youtube, pela qual tiveram acesso a um banco de questões dos tipos múltipla escolha e dissertação. Durante a atividade, os especialistas acessaram as recomendações e indicaram os resultados da avaliação em um formulário de anotações. A avaliação individual, teve duração de 1 hora para o especialista que conhecia as recomendações, e 2 horas para o outro especialista. Após a avaliação individual, os especialistas consolidaram seus resultados verificando se havia desacordo ou concordância nos problemas encontrados. Ao fim deste processo, houve a produção do relatório final da avaliação, com a descrição dos problemas da videoprova e possíveis impactos.

\section{Resultados}

Os problemas de acessibilidade para surdos encontrados no ambiente de apoio a realização do ENEM disponibilizados no Youtube são apresentados nesta seção. Alguns dos problemas identificados são apresentados de acordo com à categoria à qual pertence à recomendação não contemplada. Apesar dos problemas identificados, algumas recomendações foram atendidas e também são abordadas na seção de resultados.

Categoria: Vídeo de língua de sinais. Nesta categoria, os especialistas encontraram problemas somente com a recomendação "2. Prover vídeo de língua de sinais a todo texto escrito". Algumas informações da prova em texto no papel e também na videoprova não possuíam equivalente na língua de sinais. Um exemplo disso é a não tradução em Libras da numeração das questões, tanto em múltipla escolha como em dissertação. Outro exemplo é sobre qual alternativa da questão do tipo múltipla escolha aquela tradução em Libras se tratava, pois havia apenas uma informação em texto no canto superior esquerdo da tela. Do mesmo modo, não há tradução em língua de sinais das informações da fonte dos textos utilizados nos tipos de questões múltipla escolha e

\footnotetext{
${ }^{4}$ Youtube. https://www.youtube.com/watch?v=xh8DPkrkqSw\&list=PLjz5Kd6rxbE6AxHASITENa0uT4bZraxG
} 
VII Congresso Brasileiro de Informática na Educação (CBIE 2018)

Anais do XXIX Simpósio Brasileiro de Informática na Educação (SBIE 2018)

dissertação, como autores, título e ano. A recomendação "1. A qualidade do vídeo deve permitir que sejam captados detalhes sobre os movimentos das mãos, olhos e boca" foi contemplada, pois o vídeo disponibilizado, possui resolução que permite perceber movimentos e expressões. A recomendação "3. Fornecer mecanismo para controle da velocidade da sinalização em língua de sinais" também foi contemplada com botões para controle da velocidade do vídeo (e.g., lentidão ou rapidez).

Categoria: Apresentação alternativa para texto escrito. Os especialistas encontraram problemas com relação à recomendação "10. Videos em língua de sinais podem ser opcionalmente recuperados para cada bloco de texto" nas questões do tipo múltipla escolha e dissertativa. Os dois especialistas identificaram que o estudante não tem a possibilidade de selecionar um bloco de texto para que as informações selecionadas sejam apresentadas em vídeo de língua de sinais.

Os dois especialistas também identificaram que a recomendação " 11 . Apresentar o conteúdo em forma de texto, vídeo com a informação em língua de sinais ou um avatar traduzindo a informação para língua de sinais" não é contemplada nas questões de múltipla escolha e dissertação. A videoprova é apresentada em língua de sinais e o estudante tem acesso ao cartão resposta e a prova textual apenas em papel. Contudo, não há relação entre elas, pois o vídeo possui somente língua de sinais com algumas exceções de nomes próprios e palavras complexas e os recursos em tabelas e imagens (ver Figura 1). A videoprova não apresenta o texto concomitantemente com a língua de sinais na tela, nem oferece legenda para todas as traduções simultâneas que são apresentadas em língua de sinais. Desse modo, o estudante deve olhar ou para a tela da videoprova ou para a prova textual em papel alternadamente, sem poder visualizar ao mesmo tempo as informações.

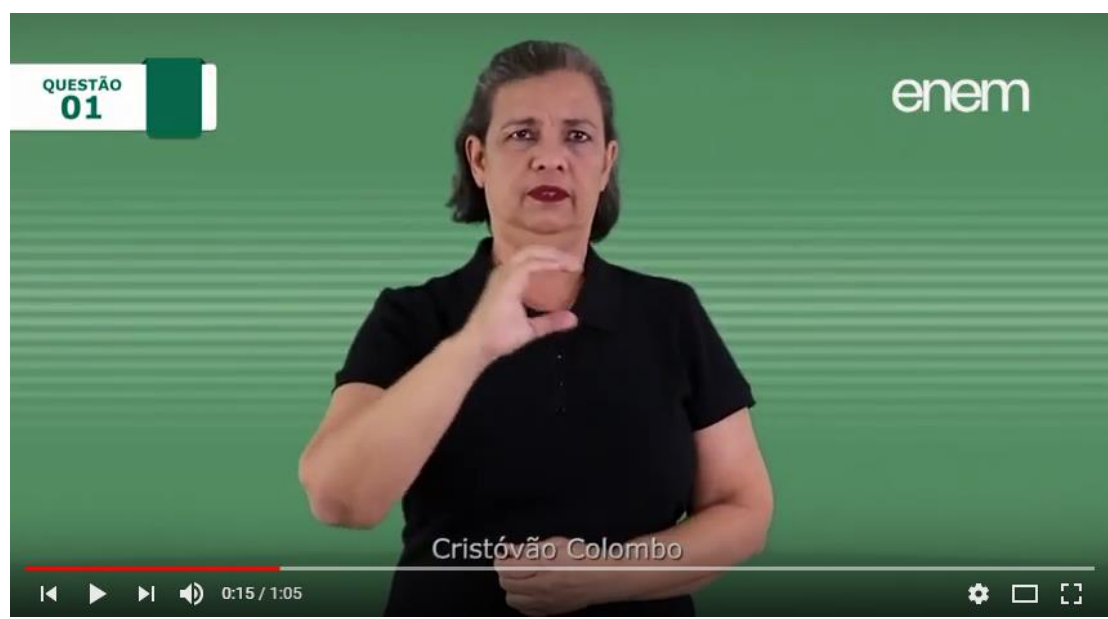

Figura 1. Exemplo do uso de textos com o vídeo em língua de sinais. Disponível em: https://youtu.be/DuoJFyLVvPA. Último acesso em 18 de setembro de 2018.

Categoria: Conteúdo. Os dois especialistas identificaram que a recomendação "20. Adicionar transcrição ao significado do conteúdo visual elou sua relação com o texto (se houver). Apesar do surdo enxergar a imagem ou vídeo, a transcrição pode ser fundamental para facilitar a sua compreensão do significado do conteúdo visual elou sua relação com o texto (se houver)" não é contemplada nos tipos de questões múltiplaescolha e dissertação. As imagens são mostradas de maneira isolada do texto e/ou língua de sinais e, ainda, não possuem transcrição, conforme ilustra a Figura 2. 
VII Congresso Brasileiro de Informática na Educação (CBIE 2018)

Anais do XXIX Simpósio Brasileiro de Informática na Educação (SBIE 2018)

Categoria: Navegação. Os especialistas identificaram que o sistema videoprova não oferece a opção de configuração de cores para nenhum tipo de questão, nem a configuração de contraste. Desse modo, o estudante não pode escolher a cor do texto nem a do fundo da tela de acordo com suas preferências. As imagens usadas na tela também não possuem qualquer tipo de cor, sendo somente apresentadas em preto e branco (ver Figura 2). Assim, os especialistas afirmaram que o sistema de apoio à realização do ENEM não contempla a recomendação "29. Configuração de cores".

$\equiv$ YouTube $^{\mathrm{BR}}$

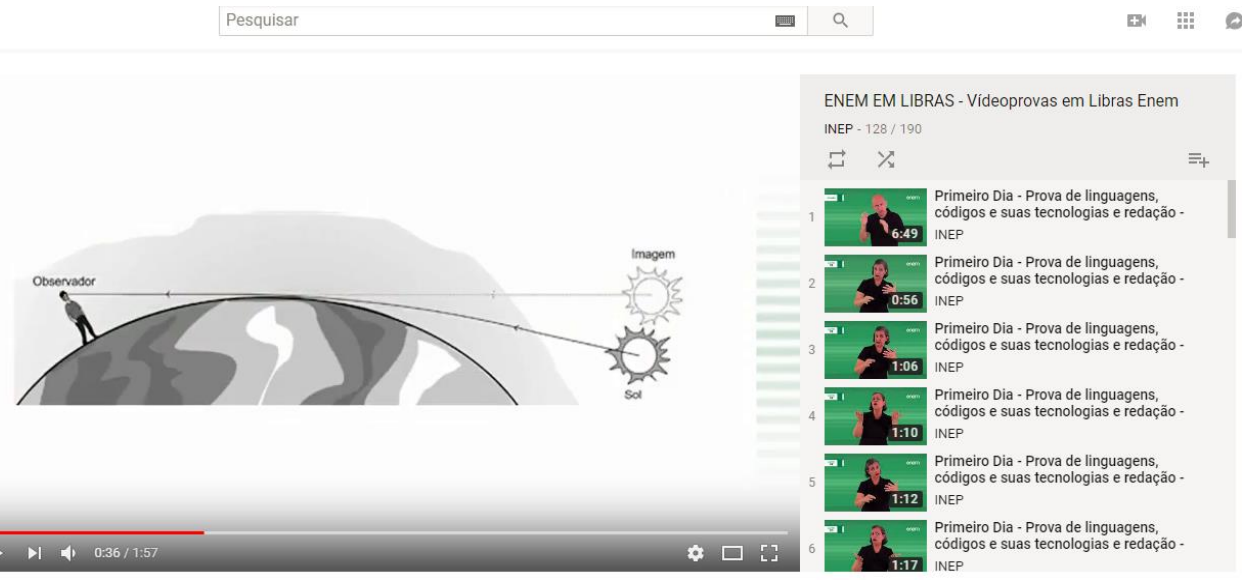

Figura 2. Imagem na tela sem recurso de transcrição. Disponível em: https://youtu.be/JDrcXOkrQ5M. Último acesso em 18 de setembro de 2018.

Ainda com relação à navegação, os especialistas identificaram que o estudante pode repetir as instruções das questões quantas vezes desejar para o tipo de questão múltipla escolha e dissertação. Dessa maneira, os especialistas concluíram que a recomendação "26. Capacidade de o estudante poder repetir as instruções quantas vezes ele desejar" foi contemplada, pois o estudante pode avançar para a questão seguinte ou voltar à questão anterior.

Os especialistas também identificaram que o sistema parecia não oferecer recursos para a modificação da interface nos tipos de questões múltipla escolha e dissertação (e.g., ampliar a interface). O vídeo em língua de sinais pode ser pausado e a sua velocidade controlada. Porém, a interface não é modificável. Não é possível controlar zoom da tela ou aumentar fontes, nem ter acesso aos gestos e expressões mais próximos, entre outras configurações. Desse modo, os especialistas afirmaram que a recomendação "32. Prover configurações para o estudante sobre o nível de ampliação (magnification) da interface de usuário" não foi contemplada.

\section{Discussão}

A avaliação realizada pelos especialistas possibilitou a identificação de informações relevantes sobre a acessibilidade dos tipos de questões do ambiente de apoio à realização do ENEM disponibilizado pelo INEP no Youtube, de maneira a mostrar que possui limitações com relação ao design da interação para os surdos. A inclusão dos estudantes surdos no ENEM passa pela solução dos problemas de acessibilidade identificados neste trabalho. Apesar de estas recomendações terem identificado problemas de acessibilidade, é possível ver que elas nos limitam a problemas referentes a aspectos técnicos da interação do usuário com a tecnologia, não abordando demais aspectos que fazem parte do mundo social no qual o usuário está inserido. 
VII Congresso Brasileiro de Informática na Educação (CBIE 2018)

Anais do XXIX Simpósio Brasileiro de Informática na Educação (SBIE 2018)

Os dois especialistas que avaliaram o sistema de apoio à realização do ENEM para surdos encontraram os mesmos problemas para os tipos de questões múltipla escolha e dissertação. Ambos especialistas identificaram também que as mesmas recomendações são contempladas nos tipos de questões múltipla escolha e no tipo de questão de dissertação, que representa a redação do ENEM. Dessa maneira, o uso das recomendações serviu de apoio para a investigação sobre os tipos de questões oferecidas pelo sistema de apoio à realização do ENEM para surdos disponibilizados pelo INEP no Youtube. Com o uso das recomendações também foi possível identificar problemas tanto relacionados ao conteúdo dos vídeos, quanto à videoprova como um ambiente interativo, o que pode indicar a importância de se pensar acessibilidade não só de sistemas interativos, mas também de seu conteúdo. Apesar disso, as recomendações não oferecem apoio para a investigação sobre aspectos da vida individual e social dos usuários, não abordando de maneira explícita o ambiente no qual estão inseridos, as demais pessoas que vivem naquele ambiente além dos usuários, os possíveis usos que a tecnologia pode apoiar bem como os efeitos que pode desencadear.

Considerando a acessibilidade da perspectiva de valores, a autonomia dos surdos é um dos exemplos de problemas que seriam identificados de maneira explícita. Os surdos têm reivindicado por anos a possibilidade do uso de tecnologia no ENEM, para que assim possam ter mais autonomia e se verem menos dependentes do intérprete. $\mathrm{O}$ atendimento diferenciado em provas de seleção é um direito de todas as pessoas com deficiência e, de acordo com o Decreto $\mathrm{n}^{0}$ 5.296/2004, as pessoas têm direito a instrumentos, equipamentos ou tecnologias adaptados. Entretanto, os recursos disponibilizados falham em prover tal autonomia, persistindo barreiras que impedirão o surdo de ter pleno acesso aos recursos necessários para realizar sua prova.

Problemas com a identidade dos surdos também podem ser observados uma vez que o sistema não disponibiliza recursos de personalização para o usuário e, assim, não respeita as possíveis diferenças relativas às proficiências em Libras e em textos em português existentes entre os surdos. Por fim, a desigualdade e a exclusão são evidenciadas uma vez que os estudantes são concorrentes ao acesso do ensino superior, e que se relacionam com questões sociais e econômicas quando se trata do ensino público. Se a tecnologia disponibilizada para apoiar os estudantes possui problemas de acessibilidade, ela pode ampliar a desigualdade de oportunidades socioeconômicas, favorecendo um grupo de pessoas enquanto exclui outro.

Além dos problemas identificados relacionados diretamente aos estudantes surdos, demais consequências negativas nas partes interessadas além do estudante surdo seriam identificadas considerando acessibilidade como um valor. Exemplos disso são: os seus familiares, que também podem ser afetados pelas questões socioeconômicas ocasionadas pelos problemas de acessibilidade identificados pelas recomendações (visão do sistema técnico), a mãe de alguns surdos, que lutam pelos direitos de igualdade de seus filhos nas escolas, o estudante surdo que pode se sentir inferior e excluído por não conseguir realizar a prova do ENEM ou por obter desempenho inferior ao esperado. Pensar em acessibilidade como um valor é considerar autonomia, igualdade e questões econômicas como requisitos adicionais às recomendações usadas neste trabalho e que transcendem questões diretas e imediatas do uso da tecnologia.

Como uma área que se preocupa com o design e avaliação de tecnologias para uso humano e com os principais fenômenos que os cercam, a IHC reconhece a necessidade de que métodos e práticas tradicionais sejam repensados com o intuito de 
VII Congresso Brasileiro de Informática na Educação (CBIE 2018)

Anais do XXIX Simpósio Brasileiro de Informática na Educação (SBIE 2018)

atender a novas demandas, como o conceito de valores humanos [Leitão et al. 2017]. Nessa direção, sugerimos que o projeto de sistemas computacionais deve ser sensível às reais necessidades da sociedade, sendo praticado a partir de uma perspectiva sistêmica e socialmente consciente, de maneira participativa e universal. De acordo com Pereira et al. (2018), um possível caminho para atender a novas demandas é repensar as teorias, métodos, ferramentas e práticas atuais de IHC com fundamentos em referencias teóricos e metodológicos que considerem além do sistema técnico, em que os valores são entendidos como características ou atributos de qualidade de uma tecnologia, também o sistema formal, cujo os valores estão conectados às normas sociais e os sistemas de leis, e o sistema informal, em que se caracterizam os valores de origem pessoal.

\section{Conclusões e trabalhos futuros}

As pesquisas em acessibilidade na Computação têm produzido resultados importantes especialmente no âmbito de tecnologias assistivas e de recomendações e padrões de acessibilidade em sistemas interativos. Embora a literatura apresente pesquisas e avanços no entendimento de acessibilidade a partir de uma perspectiva inclusiva, o entendimento e a prática em acessibilidade a partir de uma perspectiva social, beneficiando a todos, ainda é uma temática que demanda investigações e resultados.

Este artigo apresentou uma avaliação de acessibilidade nos tipos de questões oferecidas pelo ambiente de apoio a realização do ENEM para surdos disponibilizado pelo INEP no Youtube. Para a avaliação, utilizou-se um conjunto de recomendações de acessibilidade para surdos dos tipos de questões usadas na CBA em AVAs. A avaliação foi realizada por especialistas que utilizaram as recomendações para avaliar os tipos de questões múltipla escolha e dissertação com relação à compreensão do enunciado assumindo o perfil do estudante.

Os resultados da avaliação mostram problemas de acessibilidade nos tipos de questões disponibilizados pelo ambiente denominado vídeoprova no contexto dos surdos, com o uso de vídeos, textos, imagens e personalização pelo usuário. Resultados da avaliação também indicam que o uso das recomendações apoiou os especialistas na identificação de problemas de acessibilidade. Entretanto, como a literatura corrobora, as recomendações para avaliação de acessibilidade, como as adotadas para este estudo, focam somente na questão da acessibilidade em uma visão técnica na qual somente aspectos de qualidade de software são levados em consideração. O contexto social da vida dos usuários e demais partes interessadas não é abordado, de maneira que se perde capacidade não apenas de identificar problemas e suas consequências negativas, mas também de se entender as razões e necessidades de se antecipar aos problemas identificados, oferecendo soluções que efetivamente reduzam as barreiras para o acesso participativo e universal do cidadão ao conhecimento.

Como trabalhos futuros, há espaços para a elaboração de um método orientado a valores para a avaliação de sistemas computacionais capaz de identificar não somente problemas técnicos, mas também problemas com normas sociais e os sistemas de leis, e as demais questões de valor pessoal e do mundo social no qual os indivíduos vivem.

\section{Agradecimentos}

À Coordenação de Aperfeiçoamento de Pessoal de Nível Superior Brasil (CAPES) Código de Financiamento 001, o CNPq e a Alexandre Ibrahim Direne (in memoriam).

\section{Referências}


VII Congresso Brasileiro de Informática na Educação (CBIE 2018)

Anais do XXIX Simpósio Brasileiro de Informática na Educação (SBIE 2018)

Baranauskas, M.C.C. e Souza, C.S. (2006). "Desafio no 4: Acesso participativo e universal do cidadão brasileiro ao conhecimento". In: Computação Brasil, VII, n23.

Beech, M. (2011). "Computer-based testing accommodations for students with disabilities". Technical report. Ph.D. Learning Systems Institute. Florida State University. Bureau of Exceptional Education and Student Services Florida Department of Education.

Bueno, J. F., Castilho, D, F, R, J., Garcia, S. e Borrego, R. (2007). "E-learning content and adaptation for deaf students". In Proceedings 12th annual SIGCSE conference on Innovation and technology in computer science education. ACM, 271-275.

Canal, M. C. e García, L.S. (2015). "Recomendações de acessibilidade para surdos dos tipos de questões usadas na avaliação baseada em computador em ambientes virtuais de aprendizagem". In: XXVI Simpósio Brasileiro de Informática na Educação (SBIE), p. 812-821.

Canal, M. C. García, L. S. e Pereira, R. (2017). "Sistema Computacional de Apoio à Preparação para o ENEM: uma Investigação da Acessibilidade para Surdos". In: XXVIII Simpósio Brasileiro de Informática na Educação (SBIE), p. 1037-1047.

Canal, M.C. e García, L.S. (2014). "Research on Accessibility of Question Modalities Used in Computer-Based Assessment (CBA) for Deaf Education". Universal Access in Human-Computer Interaction. Universal Access to Information and Knowledge. Lecture Notes in Computer Science, Volume 8514, pp 265-276.

Gibson, J. (1979). “The Ecological Approach to Visual Perception”. Houghton Mifflin Harcourt (HMH), Boston.

Leitão, C. Maciel, C. Piccolo, L. S. G. Souza, P. C. Prates, R. O. Pereira, R. e Pereira, V. (2017). "Valores humanos em IHC: um desafio para os Grandes Desafios". In: XVI Simpósio Brasileiro sobre Fatores Humanos em Sistemas Computacionais.

Luephattanasuk, N. Suchato, A. e Punyabukkana, P. (2011). "Accessible QTI presentation for web-based elearning". In Proceedings of the International CrossDisciplinary Conference on Web Accessibility. ACM, Article 26.

Peirce, C.S. (1955). "Philosophical Writings of Peirce". Editor by J. Bachler, New York. Dover Publications.

Pereira, R. e Baranauskas, M. C. C. (2016). "Visão Sistêmica e Socialmente Consciente para Sistemas de Informação". In: Grandes Desafios em Sistemas de Informação no Brasil no Simpósio Brasileiro de Sistemas de Informação (SBSI) 2016 a 2026.

Pereira, R. Baranauskas, M. C. C. e Liu, K. (2018). “An Essay on Human Values in HCI”. SBC Journal on 3D Interactive Systems, v. 9, p. 4-16.

Pereira, R. Hayashi, E.C.S. e Baranauskas, M.C.C. (2013) "Afeto como um Valor no Design de Tecnologias Educacionais: frameworks e discussões informadas". Anais do XXIV Simpósio Brasileiro de Informática na Educação (SBIE), pp.748-757.

Santana, V.F. Melo-Solarte, D.S. Neris, V.P.A. Miranda, L.C. e Baranauskas, M.C.C. (2009). "Redes sociais online: desafios e possibilidades para o contexto brasileiro". In: XXXVI Seminário Integrado de Software e Hardware (SEMISH). 